\title{
Magnesium hydroxide whisker modified via in situ copolymerization of n-butyl acrylate and maleic anhydride
}

\author{
Yu-Zhi Jiang, Yu-Lian Wang* (10, Chun-Yan Wang, Li-Mei Bai, Xin Li, \\ Yan-Bo Li
}

Received: 28 September 2015/Revised: 3 November 2015/ Accepted: 8 November 2016/Published online: 3 March 2017

(C) The Author(s) 2017. This article is published with open access at Springerlink.com

\begin{abstract}
Magnesium hydroxide (MH) whiskers were modified via in situ polymerization of n-butyl acrylate and maleic anhydride. Sodium dodecyl sulfonate was used as emulsifier. The modifying effect was evaluated by using contact angle and activation index. The thermal stability, functional groups, structure, morphology, phase composition and surface element valence of $\mathrm{MH}$ whiskers were characterized by thermogravimetry-differential scanning calorimetry (TG-DSC), Fourier transform infrared spectroscopy (FTIR), X-ray diffraction (XRD), scanning electron microscopy (SEM) and X-ray photoelectron spectroscopy (XPS). Results reveal that the contact angle and activation index of modified $\mathrm{MH}$ whiskers are $105^{\circ}$ and $76.5 \%$, the thermal stability shows little change, and the decomposition temperature ranges between 38 and $419{ }^{\circ} \mathrm{C}$. The copolymer of n-butyl acrylate and maleic anhydride absorbed on the surface of $\mathrm{MH}$ whiskers leads to the increased diameter and makes the surface of whiskers be rougher. Furthermore, the absorption of element $\mathrm{C}$ on the surface of $\mathrm{MH}$ whiskers increases, and the diffraction
\end{abstract}

Y.-Z. Jiang, Y.-L. Wang*, X. Li

Collogy of Materials Science and Engineering, Shenyang Ligong

University, Shenyang 110159, China

e-mail: xinxinwyl@163.com

C.-Y. Wang

Kailong Lanfeng New Material Technology Co., Ltd,

Zhenjiang 212132, China

L.-M. Bai

College of Mining Engineering, Hebei United University,

Tangshan 063009, China

Y.-B. Li

School of Materials Science and Engineering, Central South University, Changsha 410083, China intensity of $\mathrm{C} 1 \mathrm{~s}$ spectra increases; thus, the compatibility of whiskers in the organic phase can be improved significantly. Lastly, the surface molecular model of MH whiskers modified via in situ copolymerization of n-butyl acrylate and maleic anhydride is established.

Keywords Magnesium hydroxide whiskers; n-Butyl acrylate; Maleic anhydride; In situ copolymerization; Surface molecular model

\section{Introduction}

Magnesium hydroxide (MH) whiskers, a kind of functional single crystal with smooth and slim appearance, have attracted considerable attention [1]. MH whiskers are usually used as a filler of polymers to improve their property of reinforcement, flame retardant and smoke suppression, because of their excellent characteristics such as better toughening and reinforcing performances [2, 3]. However, $\mathrm{MH}$ whiskers are easy to agglomerate in the preparation and processing for their high surface energy. This property leads to a poor dispersion for $\mathrm{MH}$ whiskers in polymer material. Furthermore, the surface of MH whiskers is hydrophilic, which also results in weak combination between MH whiskers and polymer matrix [4-6]. These problems make the composites rarely meet the actual requirement on mechanical properties. Therefore, in order to enhance the compatibility with polymers, the surface modification of $\mathrm{MH}$ whiskers is quite important. In recent years, there are an increasing number of studies on the improvement in the compatibility between additive materials and polymer matrix [7, 8]. The activity and coupling agent mostly used in surface modification of additive material have obtained significant effects. The modification 
of $\mathrm{MH}$ whiskers has been broadly investigated, and the involved surfactants mainly include silanes, titanates, aluminate esters, etc. [9-12].

In this paper, $\mathrm{MH}$ whiskers were modified via in situ copolymerization of n-butyl acrylate and maleic anhydride. The modification effect was characterized by evaluation of wettability. The thermal stability, functional groups, structure, morphology, phase composition and surface element valence of $\mathrm{MH}$ whiskers both modified and unmodified were characterized, and the surface molecular model of modification of $\mathrm{MH}$ whiskers was established.

\section{Experimental}

\subsection{Preparation of MH whiskers}

$\mathrm{MH}$ whiskers were prepared via hydrothermal synthesis method using precursor of magnesium oxysulfate (MOS) whiskers and $\mathrm{NaOH}$ as reactants. The initial (MOS) whiskers slurry with a concentration of $4 \mathrm{wt} \%$ was prepared, and $1.0 \mathrm{~mol} \cdot \mathrm{L}^{-1} \mathrm{NaOH}$ was added to the slurry in the mole ratio of $4.5: 1.0(\mathrm{NaOH} / \mathrm{MOS})$. The well-mixed solution was put into the GSH-10 magnetic agitated autoclave under the stirring speed of $100 \mathrm{r} \cdot \mathrm{min}^{-1}$. The reaction was carried out at $180{ }^{\circ} \mathrm{C}$ for $4 \mathrm{~h}$. When the reactor cooled down to room temperature at the end of the reaction, the product was dispersed, filtrated and dried.

\subsection{Modification of $\mathrm{MH}$ whiskers}

All chemicals used were of analytical grade (Tian-jin Kemiou Chemical Reagent Co., Ltd.) and used without further purification. Water used was deionized water. $3.75 \mathrm{~g}$ n-butyl acrylate and $50 \mathrm{ml}$ sodium dodecyl sulfonate solution (concentration of $15 \mathrm{wt} \%$ ) were mixed in a three-mouth flask and kept stirring, and $15 \mathrm{~g} \mathrm{MH}$ whiskers was added gradually. The heating temperature was increased to $60-70{ }^{\circ} \mathrm{C}$, and then, $62.5 \mathrm{ml}$ aqueous solution of maleic anhydride (concentration of $2 \mathrm{wt} \%$ ) was added slowly within $30 \mathrm{~min}$. The composite was kept reacting for $2 \mathrm{~h}$ under the temperature of $85^{\circ} \mathrm{C}$. After the demulsification, the suspended emulsion was washed, filtered and dried. Finally, the modified MH whiskers were obtained.

\subsection{Characterization}

GB/T16913.5-1997 standard was applied to test the contact angle of $\mathrm{MH}$ whiskers. According to the activation index method, $10 \mathrm{~g}$ modified $\mathrm{MH}$ whiskers was added to $100 \mathrm{ml}$ deionized water under stirring for 2 min and then kept still for a certain time till the solution was clarified. Then, the $\mathrm{MH}$ whiskers floating on the surface of the solution were taken out. And the $\mathrm{MH}$ whiskers precipitated at the bottom of the beaker were filtered, dried and weighed. The ratio of floating $\mathrm{MH}$ whiskers to the total modified $\mathrm{MH}$ whiskers used was defined as the activation index. The formula for activation index is shown below:

$H=\frac{m-m_{1}}{m} \times 100 \%$

where $H(\%)$ stands for activation index, $m(\mathrm{~g})$ represents the total mass of $\mathrm{MH}$ whiskers added, and $m_{1}(\mathrm{~g})$ stands for the mass of $\mathrm{MH}$ whiskers precipitated at the bottom of the beaker.

Thermal stability of the modified and unmodified $\mathrm{MH}$ whiskers products was investigated via thermogravimetrydifferential scanning calorimetry (TG-DSC, SDT 2960 TA USA) at a heating rate of $10{ }^{\circ} \mathrm{C} \cdot \mathrm{min}^{-1}$ under an air atmosphere. The functional groups of the products were analyzed by Fourier transform infrared spectroscopy (FTIR, 380, Nicolet Company). The phase compositions of the products were analyzed by X-ray diffractometer (XRD, Ultima IV, Rigaku, Japan, $40 \mathrm{~mA}, 40 \mathrm{kV}$ ) with $\mathrm{Cu} \mathrm{K \alpha}$ radiation at scanning rate of $8\left(^{\circ}\right) \cdot \mathrm{min}^{-1}$ from $10^{\circ}$ to $90^{\circ}$. The wavelength was $1.54056 \mathrm{~nm}$, the stability of current was $\pm 0.03 \%$, and the accuracy of $\theta$ was $\pm 0.002^{\circ}$. The morphology of the products was analyzed by scanning electron microscopy (SEM, S-3400N, Hitachi, Japan) at an accelerating voltage of $0.50-30.00 \mathrm{kV}$, a beam current of $1 \mathrm{pA}-1 \mu \mathrm{A}$ and a resolution of $1.0 \mathrm{~nm}$ after the samples were coated with gold. The dried products were examined by X-ray photoelectron spectroscopy (XPS, ESCALAB250, Hitachi, Japan) to study the bonding energy of $\mathrm{Mg}, \mathrm{O}, \mathrm{C}$ elements and valence change in whiskers' surface elements with an emitter of $\mathrm{Al} \mathrm{K} \alpha$, power of $150 \mathrm{~W}$ ( $h v=1486.6 \mathrm{eV}, I=10 \mathrm{~mA})$, spot size of $500 \mu \mathrm{m}$, transmission energy of $50 \mathrm{eV}$ and energy step of $0.1 \mathrm{eV}$.

\section{Result and discussion}

\subsection{Contact angle and activation index of $\mathrm{MH}$ whiskers} before and after modification

Table 1 shows contact angle and activation index of $\mathrm{MH}$ whiskers before and after modification. The MH whiskers modified via in situ polymerization of n-butyl acrylate and maleic anhydride achieve a contact angle of $105^{\circ}$ (contact

Table 1 Contact angle and activation index of MH whiskers before and after modification

\begin{tabular}{lcc}
\hline Types of $\mathrm{MH}$ & Contact angle $\left./{ }^{\circ}\right)$ & Activation index $/ \%$ \\
\hline MH before modification & 1.30 & 0.16 \\
MH after modification & 105.00 & 76.50 \\
\hline
\end{tabular}


angle before modification is $1.30^{\circ}$ ) and an activation index of $76.5 \%$ (activation index before modification is $0.16 \%$ ). Obviously, the modification has a significant impact on MH whiskers.

\subsection{TG-DSC analysis of MH whiskers before and after modification}

TG-DSC curves for MH whiskers before and after modification are shown in Fig. 1. As seen in Fig. 1, compared with unmodified $\mathrm{MH}$ whiskers, modified $\mathrm{MH}$ whiskers have decomposition temperature ranging from 382 to $419{ }^{\circ} \mathrm{C}$. TG curves show a declined trend when the temperature falls below $382{ }^{\circ} \mathrm{C}$. It is caused by the thermal decomposition of a small amount of copolymer modifier. The complete decomposition temperature of $\operatorname{Mg}(\mathrm{OH})_{2}$ is $490{ }^{\circ} \mathrm{C}$ in theory, yet neither of the composites is decomposed completely after the temperature increases above $490{ }^{\circ} \mathrm{C}$. It is due to the fact that a trace amount of heatresistant decomposition products is mixed in the course of the experiment [13]. Thus, the copolymer film improved the dispersibility and compatibility of whiskers, yet it has little effect on thermal stability.

\subsection{FTIR analysis of $\mathrm{MH}$ whiskers}

before and after modification

Figure 2 shows FTIR spectra of MH whiskers. Compared with unmodified whiskers, the significant decrease in $-\mathrm{OH}$ stretching vibration peaks at $3695 \mathrm{~cm}^{-1}$ and the sharper and wider absorption peak at $3434 \mathrm{~cm}^{-1}$ is attributed to the association of more $-\mathrm{OH}$ for modified $\mathrm{MH}$ whisker. The characteristic absorption peaks at 2962, 2869 and $1382 \mathrm{~cm}^{-1}$ are ascribed to the asymmetric vibration, symmetric vibration and symmetric deformation vibration of $-\mathrm{CH}_{3}$, respectively. Moreover, the absorption peaks at 2915 and $2844 \mathrm{~cm}^{-1}$ are assigned to the asymmetric vibration and symmetric vibration of $-\mathrm{CH}_{2}$. The sharp peak at $1729 \mathrm{~cm}^{-1}$ corresponds to $\mathrm{C}=\mathrm{O}$ vibration of ester. The

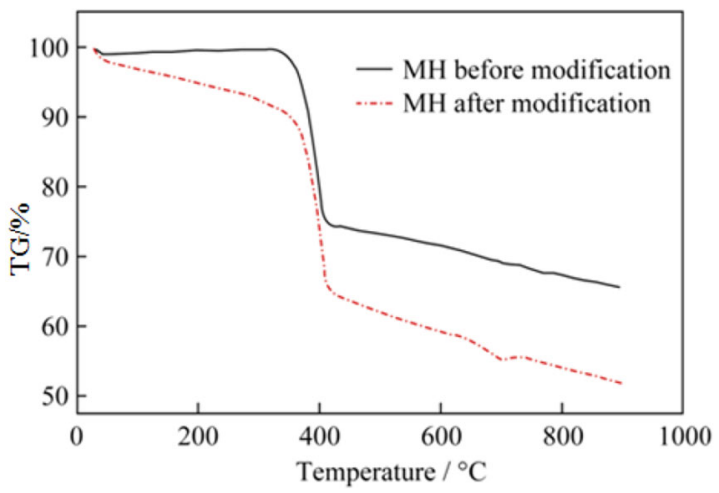

Fig. 1 TG spectra of MH whiskers before and after modification

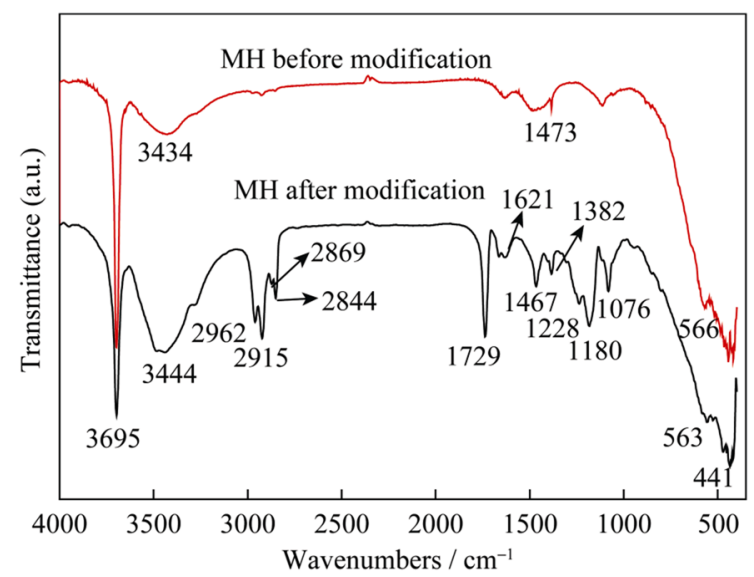

Fig. 2 FTIR spectra of MH whiskers before and after modification

small absorption peaks at 1621 and $1467 \mathrm{~cm}^{-1}$ are the asymmetric vibration and symmetric vibration of - $\mathrm{COO}-$, respectively. The peaks at $1228 \mathrm{~cm}^{-1}$ are assigned to the stretching vibration of $\mathrm{C}-\mathrm{O}$ of carboxylic acid. The peaks at 1180 and $1076 \mathrm{~cm}^{-1}$ are ascribed to $\mathrm{C}-\mathrm{O}$ groups of the ester. Finally, the absorption peaks below $566 \mathrm{~cm}^{-1}$ are the stretching vibration of $\mathrm{Mg}$.

Figure 2 provides the evidence for the existence of copolymers of n-butyl acrylate and maleic anhydride on the surface of modified $\mathrm{MH}$ whiskers. The coordination happens between carboxylate radical of copolymers and magnesium of $\mathrm{MH}$ whiskers, and the coordination pattern is affected by the location of antisymmetric and symmetric stretching vibration frequency of carboxylate radical. The frequency difference in the monodentate ligands is more than $200 \mathrm{~cm}^{-1}$, while it is around $150 \mathrm{~cm}^{-1}$ for bridged coordination [14]. However, only slight differences exist among dozens of wavenumber in bidentate ligands. As seen in Fig. 2, the corresponding asymmetric vibration and symmetric vibration of stretching absorption peaks of COO- are 1621 and $1467 \mathrm{~cm}^{-1}$, respectively. The discrepancy of $154 \mathrm{~cm}^{-1}$ between them proves that bridged coordination is formed between magnesium and carboxylate radical.

\subsection{XRD analysis of MH whiskers before and after modification}

Figure 3 represents phase composition of $\mathrm{MH}$ whiskers before and after modification. It can be seen that the diffraction peaks of modified $\mathrm{MH}$ whiskers are consistent with the standard diffraction pattern of $\mathrm{Mg}(\mathrm{OH})_{2}$, and no other peaks exist. It is illustrated that the crystals of modified $\mathrm{MH}$ whiskers do not change, and the purity remains at a high level [15]. However, compared with modified $\mathrm{MH}$ whiskers, for unmodified $\mathrm{MH}$ whiskers, the shape of the diffraction peaks is sharper and the intensity is 


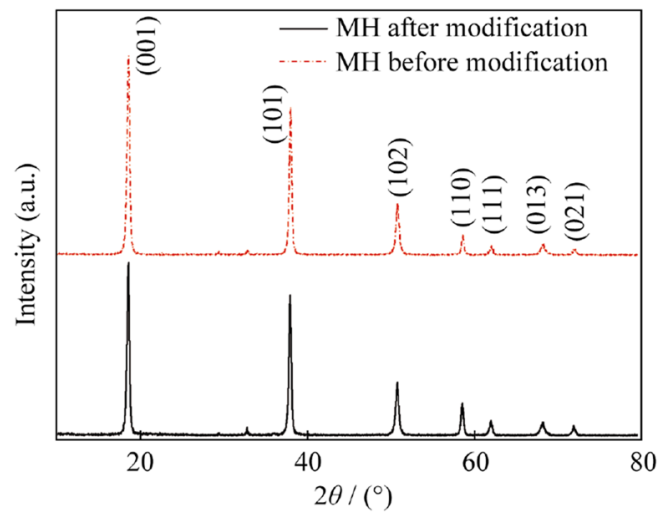

Fig. 3 XRD patterns of MH whiskers before and after modification

slightly higher, indicating that the copolymer particulates on the surface of MH whiskers weaken the intensity of diffraction peaks and prevent agglomeration of whiskers.

\subsection{Surface morphology of MH whiskers}

before and after modification

Figure $4 \mathrm{a}$ shows that the morphology of unmodified $\mathrm{MH}$ whiskers is straight, slim and easily agglomerated with smooth surface, while the modified MH whiskers are coated by spot and film-like material, as shown in Fig. 4b. It suggests that a copolymer layer made of n-butyl acrylate and maleic anhydride is generated [16]. The layer makes the surface of modified MH whiskers be rougher and improves the dispersion of whiskers significantly.

\subsection{XPS analysis of MH whiskers}

before and after modification

Figure 5 shows that $\mathrm{Mg}$ and $\mathrm{O}$ are present on the surface of unmodified $\mathrm{MH}$ whiskers, while element $\mathrm{C}$ is found after modification. Comparisons of the spectra for $\mathrm{MH}$ whiskers before and after modification show that the peaks for elements $\mathrm{C}$ and $\mathrm{O}$ are significantly higher, suggesting that the copolymer containing element $\mathrm{C}$ is generated on the

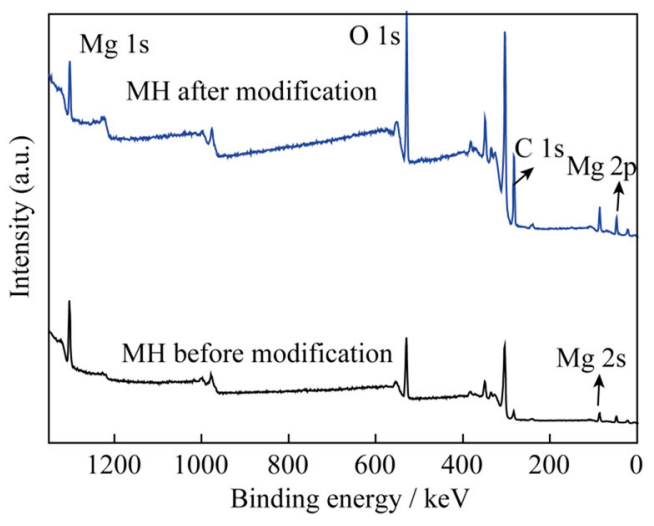

Fig. 5 XPS spectra for surface element analysis of MH whiskers before and after modification

surface of whiskers after modification. It also confirms that the particulates in Fig. 1b are the copolymer of n-butyl acrylate and maleic anhydride. In order to further study the changes in the spectra for the elements, the peaks are dealt separately. The spectra for $\mathrm{C} 1 \mathrm{~s}, \mathrm{O} 1 \mathrm{~s}$ and $\mathrm{Mg} 1 \mathrm{~s}$ are obtained and analyzed, and the results are shown in Fig. 6.

As shown in Fig. 6a, $\mathrm{Mg}$ 1s binding energies of modified and unmodified $\mathrm{MH}$ whisker are, respectively, 1302 and $1303 \mathrm{eV}$, indicating that negative chemical displacement takes place and the electron cloud density of $\mathrm{Mg}$ atom increases after $\mathrm{MH}$ whiskers were modified. It is evident from Fig. $6 \mathrm{~b}$ that no significant negative chemical displacements are found, while $\mathrm{O} 1 \mathrm{~s}$ peak intensity of modified $\mathrm{MH}$ whisker is strengthened, which could be attributed to the layer generated on the surface of whiskers after modification. However, apparent $\mathrm{C} 1 \mathrm{~s}$ spectra appearing in Fig. $6 c$ could be found after modification, confirming the presence of organic layer on the surface of modified $\mathrm{MH}$ whisker. Moreover, the thickness of the layer is positively related to the peak intensity of elements [17]. Therefore, the thickness of the layer can be determined according to the peak intensity of elements. The peak intensity of $\mathrm{C} 1 \mathrm{~s}$ spectra is very sharp after modification, demonstrating that $\mathrm{C}$ elements are adsorbed on the surface of modified $\mathrm{MH}$
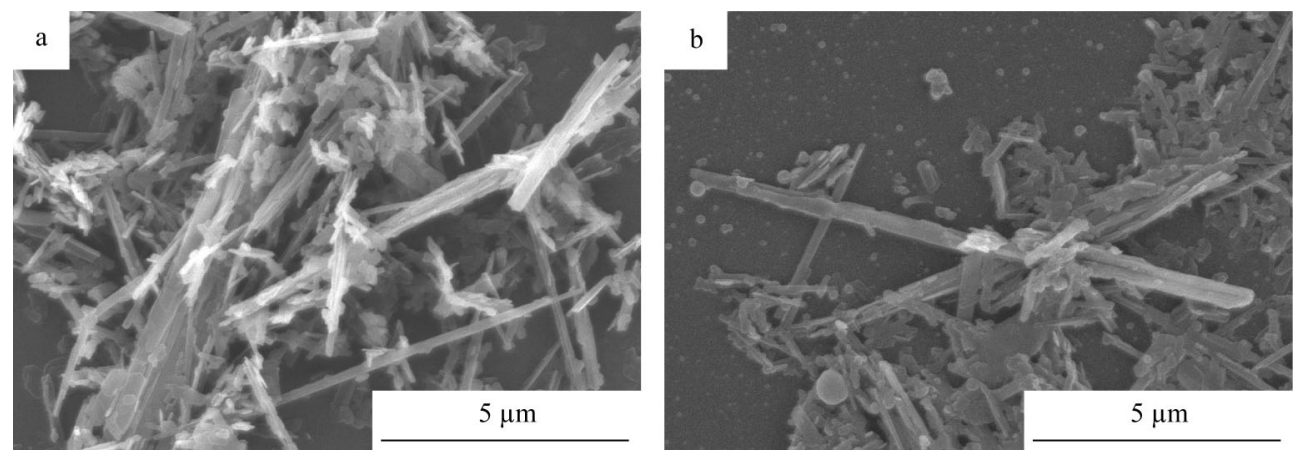

Fig. 4 SEM images of MH whiskers: a MH whiskers before modification and $\mathbf{b}$ MH whiskers after modification 

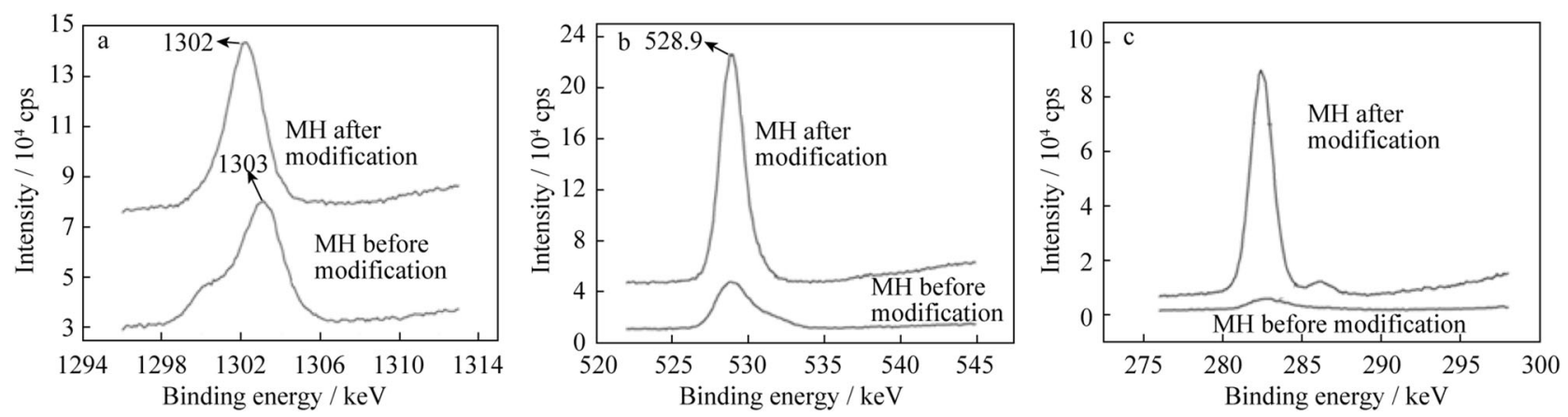

Fig. 6 XPS spectra for peak contrast of single element on MH whiskers before and after modification: a Mg 1 s, b O 1s and c C 1s
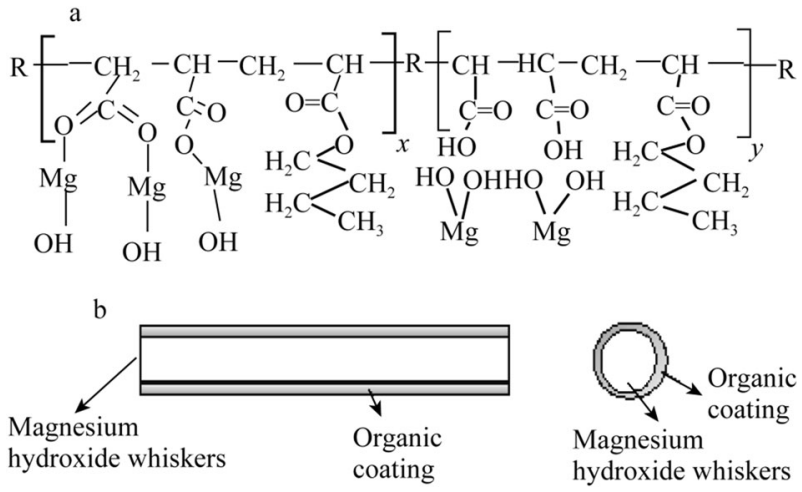

Fig. $7 \mathrm{MH}$ whiskers modified via in situ copolymerization: a microstructure of organic coating and $\mathbf{b}$ macrostructure of modified magnesium hydroxide whiskers

whiskers. However, there are no C 1s spectra in unmodified MH whiskers.

\subsection{Surface molecular model of modified MH whiskers}

Based on FTIR, SEM and XPS analysis, the surface molecular model of $\mathrm{MH}$ whiskers modified via in situ copolymerization can be demonstrated.

In Fig. 7, the polymerizable small molecule monomers are coordinated with magnesium ions and hydroxyl on the surface of MH whiskers. While the initiator was added to micro-emulsion, the small molecule monomers are copolymerized and an organic coating is formed on the surface of MH whiskers. The formation of the copolymer can be verified by FTIR analysis of functional groups. Meanwhile, the thickness of copolymers could be determined by the peak height of C 1s spectra on the surface of modified MH whiskers. Based on the results of the analysis, the structure model of modified $\mathrm{MH}$ whiskers is established, which is consistent with the functional group analysis and elemental analysis, and this confirms the rationality and accuracy of the structure model.

\section{Conclusion}

MH whiskers were modified successfully via in situ copolymerization of n-butyl acrylate and maleic anhydride. The contact angle and activation index of modified $\mathrm{MH}$ whiskers are, respectively, $105^{\circ}$ and $76.5 \%$. The thermal stability of $\mathrm{MH}$ whiskers decreases slightly due to the existence of the copolymer, but the compatibility of whiskers in the organic phase is improved significantly after modification. SEM and XRD results show that a lot of copolymer particulates are generated on the surface of $\mathrm{MH}$ whiskers, which makes it rough and weakens the intensity of diffraction peaks. According to XPS analysis, the decrease in binding energy of $\mathrm{Mg}$ element induced the negative chemical displacement, and the modifier molecular is coordinated with $\mathrm{Mg}$ element on the surface of $\mathrm{MH}$ whiskers. Based on analysis results, the surface molecular model of MH whiskers modified via in situ copolymerization of n-butyl acrylate and maleic anhydride is established.

Acknowledgements This study was financially supported by the National Natural Science Foundation of China (No.51272163).

Open Access This article is distributed under the terms of the Creative Commons Attribution 4.0 International License (http:// creativecommons.org/licenses/by/4.0/), which permits unrestricted use, distribution, and reproduction in any medium, provided you give appropriate credit to the original author(s) and the source, provide a link to the Creative Commons license, and indicate if changes were made.

\section{References}

[1] Sun HM, Sun QJ, Li W, Dai L, Zhao GL. Research progress on the application of domestic inorganic whiskers in polymer composites. Fine Spec Chem. 2010;18(9):9.

[2] Wu JS, Xiao YK, Chen LY, Chen XW. Synthesis of magnesium hydroxide whisker via glycerol-inverter microwave-hydrothermal method. Chem J Chin Univ. 2009;30(12):2354.

[3] Wang P, Li C, Gong H, Wang H, Liu J. Morphology control and growth mechanism of magnesium hydroxide nanoparticles via a simple wet precipitation method. Ceram Int. 2011;37(8):3366. 
[4] Jiang YZ, Wang CY, Xu ZS, Zhang ZY. Surface modification mechanism of magnesium oxysulfate whiskers via wet chemical method. Rare Met. 2016;35(11):874.

[5] Huang QJ, Liao MC, Zeng HY, Zhang W, Zhang ZQ, Liu XJ, Li $\mathrm{H}$. Effects of surface pretreatment on structure and release performances of $\mathrm{Mg}-\mathrm{Al}$ hydrotalcites. Chin J Inorg Chem. 2014; 30(7): 1542.

[6] Wang HC, Yan ZR, Deng L, Dong H, Jia YH, Ma N, He J, Yu GH. Synthesis and surface modification of cobalt nanoparticles and electromagnetic property of $\mathrm{Co} / \mathrm{PPy}$ nanocomposites. Rare Met. 2015;34(4):223.

[7] Liu SP, Chen J, Tan KJ, Zhang M, Zhou XP. Rheological properties of HIPS/modified magnesium hydroxide whiskers composites. J Wuhan Inst Technol. 2009;31(7):18.

[8] Braun U, Schartel B. Flame retardant mechanisms of red phosphorus and magnesium hydroxide in high impact polystyrene. Macromol Chem Phys. 2004;205(16):2185.

[9] Zhen XG, Li CH, Li HM. Research on the industrial-grade magnesium hydroxide modificated by hydrothermal method. J Salt Lake Res. 2010;18(3):68.

[10] Wu JS, Kuang WH, Liang HQ. Progress in the development of magnesium hydroxide flame retardant. J Salt Chem Ind. 2007; 37(2):35.
[11] Zhou ZZ, Deng YL. Solution synthesis of magnesium hydroxide sulfate hydrate nanobelts using sparingly soluble carbonate salts as supersaturation control agents. J Colloid Interface Sci. 2007; 316:185.

[12] Sun XT, Li X. Hydrothermal conversion of magnesium oxysulfate whiskers to magnesium hydroxide nanobelts. Mater Chem Phys. 2008;109:381.

[13] Xu JF, Shi W, Ni ZM, Sun ZY, Qian PP. Surface characterization for anionic-nonionic surfactant modified layered double hydroxides. Chin J Inorg Chem. 2014;30(5):977.

[14] Weng SF. Analysis of Fourier Transform Infrared Spectroscopy. Beijing: Chemical Industry Press; 2010. 306.

[15] Zhang J, Bao FR, Dai DP, Zhou NL, Li L, Lu S, Shen J. Preparation and surface characterization of the nano-calcium carbonate modified with maleic anhydride. Chin J Inorg Chem. 2007;23(5):823.

[16] Dong HB, Du ZP, Zhao YH, Zhou DP. Preparation and properties of modified nano magnesium hydroxide. Chin Powder Technol. 2010;16(4):58.

[17] Gu XY, Wu XD. Research progress on the surface modification of magnesium hydroxide flame retardant. Anhui Chem. 2011; 37(6):5. 